Mots. Les langages du politique

70 | 2002

La politique en chansons

\title{
Entretien avec Magid Cherfi, Zebda
}

Marlène Coulomb-Gully

\section{OpenEdition}

Journals

Édition électronique

URL : https://journals.openedition.org/mots/9773

DOI : $10.4000 /$ mots. 9773

ISSN : 1960-6001

\section{Éditeur}

ENS Éditions

\section{Édition imprimée}

Date de publication : 1 novembre 2002

Pagination : 125-131

ISBN : 2-84788-016-X

ISSN : 0243-6450

\section{Référence électronique}

Marlène Coulomb-Gully, "Entretien avec Magid Cherfi, Zebda », Mots. Les langages du politique [En ligne], 70 | 2002, mis en ligne le 07 mai 2008, consulté le 22 avril 2022. URL : http:// journals.openedition.org/mots/9773; DOI : https://doi.org/10.4000/mots.9773 


\section{Marlène COULOMB-GULLY ${ }^{\circ}$}

\section{Entretien avec Magid Cherfi, Zebda}

Composé de sept membres, trois Kabyles de seconde génération, trois Gascons des Pyrénées et un Parisien, Zebda est un groupe musical toulousain connu pour son engagement politique radicalement à gauche. Magid Cherfi en est le chanteur et l'auteur principal des textes. L'histoire de Zebda confond le groupe, à ses débuts au milieu des années quatrevingt, avec l'association socio-culturelle Vitécri, qui œuvre dans les quartiers Nord de Toulouse. En 1997, il contribue à créer le Tactikollectif, association en pointe dans nombre de combats sociaux et politiques (sans-papiers, double-peine, etc.). Plus récemment, il a initié la dynamique de la liste Motivé-e-s, qui a obtenu 12,8\% des voix au premier tour des dernières élections municipales toulousaines.

En 1992, le premier album de Zebda se signale par sa pochette qui montre un enfant de l'Intifada, une fronde à la main. Son second album, paru en 1995, s'intitule "Le bruit et l'odeur», en réponse à une phrase de J. Chirac stigmatisant les «étrangers ${ }^{1}$. Motivés, sous-titré Chants de lutte, parait en 1997 et tente de répondre au besoin d'indépendance financière du Tactikollectif; le succès est immédiat, puisque très vite, sans publicité ni promotion, ce sont 150000 disques qui sont vendus. "Tomber la chemise ", extrait de l'album Essence ordinaire, remporte en 1999 deux victoires de la musique. La sortie du prochain album est prévue à l'automne 2002.

- Laboratoire d'Études et de Recherches Appliquées aux Sciences Sociales (LERASS), 115 Route de Narbonne, 31077 Toulouse Cedex 4.

1. «Si vous ajoutez à cela les allocations perçues par les étrangers, le bruit et l'odeur, le travailleur français, sur le palier, il devient fou », J. Chirac, 1991. 
Mots : L'album Motivés, et plus particulièrement Le chant des partisans, avec le refrain «Motivés, motivés, il faut rester motivés » que vous $y$ avez adjoint, vous a fait connaitre du grand public. Qu'est-ce qui vous a conduits à cet album de "chants de lutte », comme vous le qualifiez?

M. Cherfi : Précisons tout d'abord qu'il y a eu pour les gens un amalgame entre l'album Motivés et le groupe «Zebda», les gens nous connaissant plus dans notre dimension sociale et politique qu'esthétique ${ }^{2}$. On a bâti notre histoire sur nos valeurs, sur nos idées plus que sur l'innovation artistique : nous sommes de piètres créateurs au regard de ce qui est novateur sur le plan artistique.

On est montés sur scène pour les mêmes raisons que celles qui nous ont fait entrer en politique ou qui nous ont conduits à chanter. Nous avons une histoire d'enfants de l'immigration, nous sommes fils d'immigrés et tout est centré là-dessus, dans notre quotidien, dans notre vision des choses... C'est cela qui nous a amenés à nous montrer, à manifester dans la rue, à publier quelque chose, pour dire " on est là ». Et cette nécessité s'est exprimée par la chanson parce que c'était pour nous ce qu'il y avait de plus simple, de plus immédiat : il suffisait d'un bar, d'une batterie, d'une voix.

On devait nécessairement en arriver à l'album Motivés, à ce recueil de chants révolutionnaires, à quelque chose de $100 \%$ politique. Toute notre histoire nous a conduits vers ça.

\section{Mots : Pourquoi la LCR a-t-elle financé cet album?}

M. Cherfi : On a toujours flirté avec les mouvements de gauche et d'extrême-gauche ${ }^{3}$, on a toujours été fascinés par ces gens qui donnent leur vie à un idéal, à une cause. Ils disent " On va les défendre » là où nous on a commencé par dire «On veut se défendre, on veut qu'on nous défende ». Cet engagement pour les autres est fascinant. Et puis, le côté « extrême » a quelque chose de subversif, d'excitant. Et on a toujours eu des copains à la Ligue, au PS et au PC.

2. Ce sont en fait trois chanteurs de Zebda ainsi qu'un collectif de musiciens qui ont participé à l'album Motivés.

3. Au premier tour de l'élection présidentielle, Motivés a appelé à voter pour Olivier Besancenot, candidat de la Ligue Communiste Révolutionnaire (trostskyste). Au second tour, le groupe Zebda a fait partie des artistes réunis à Paris pour appeler à voter contre l'extrême-droite de Le Pen et du FN. 
Mots : Qu'est-ce qui, dans l'album Motivés, a présidé au choix de ces chants révolutionnaires et pas d'autres? Pourquoi Le chant des partisans, par exemple, et pas La Marseillaise ${ }^{4}$ ?

M. Cherfi : Il fallait une certaine diversité, une certaine représentativité dans l'espace, dans le temps : d'où des chants français, italiens, espagnols, contemporains, mais aussi des siècles passés.

Pour nous, La Marseillaise, c'est la France, un pays qui, d'une certaine façon, ne veut pas de nous. Dans Le chant des partisans, ce qui est saillant, c'est la notion de résistance, de résistance au fascisme, beaucoup plus que l'exaltation de la nation ou d'un quelconque sentiment patriotique.

Mots : C'est le seul chant de l'album que vous ayez recomposé, avec un nouveau refrain, une nouvelle ligne mélodique : pourquoi?

M. Cherfi : Il fallait en atténuer le côté dramatique, y insuffler quelque chose de gai, de dynamique, qui rassemble les troupes. C'était l'objectif de l'album : rassembler les troupes. Et le mot motivé est venu là, c'est Mous qui l'a trouvé 5 .

Mots : Motivés : c'est un participe passé passif, que vous employez sans complément d'agent... Vous êtes " motivés 》 dans l'absolu, et pas motivés "par » quelque chose de particulier : est-ce à dire que vous prônez l'engagement en soi, indépendamment de la cause qui suscite cet engagement, comme si l'objet de l'engagement était secondaire?

M. Cherfi : Non, bien sûr que non... Mais c'est vrai que notre action au Tactikollectif, à Vitécri, la lutte et l'engagement au quotidien nous ont peut-être conduits à une lucidité proche d'un certain cynisme, qui nous empêche de croire à un idéal. Et pourtant il en faut un.

C'est un peu à l'image du mouvement des Motivé-e-s : on se jette dans la bataille politique en se disant qu'on n'a rien à y faire et pourtant on $\mathrm{y}$ va. On sait qu'on ne changera pas la société, mais il faut essayer. Il faut qu'on réfléchisse, qu'on agisse, qu'on soit conscient des choses, tout en

4. L'album Motivés comprend les chants suivants : 1. Motivés, Le chant des partisans 2. El paso del Ebro 3. Hasta siempre 4. Nekwni s warrach n lezzayer 5. Bella ciao 6. La butte rouge 7 . La cucaracha 8 . Bandiera rossa 9. Le temps des cerises 10. Nicaraguita 11. L'estaca.

5. Moustapha Amokrane, dit « Mous », musicien de Zebda. 
acceptant que ça ne serve pas à grand chose. Le mot motivé, avec sa force, permet de compenser le doute.

Mots : Dans Le chant des partisans, ce que tout le monde a retenu, c'est le refrain "Motivés, motivés, il faut rester motivés... ». Est-ce qu'il n'y a pas là un vrai malentendu, qui renverrait plus largement à un malentendu concernant la chanson politique dans son ensemble: on en retient quelque chose qui ne renvoie pas nécessairement à l'engagement, mais au seul côté entrainant, sympathique de la chanson. L'objectif atteint n'est-il pas loin de l'objectif visé?

M. Cherfi : Je suis d'accord avec vous. Il y a quelques jours, j'ai rencontré Vincent Lagaffe ${ }^{6}$, qui s'est mis à me chanter "Motivés, motivés...» et je me suis dit «Quel échec ... ». Mais la chanson politique est aussi une chanson : ça doit donc être un moment agréable à passer. Si c'est un discours de Georges Marchais mis en musique, ce n'est plus une chanson et ça n'a plus d'intérêt.

Mots : Quelle est selon vous la fonction de la chanson politique?

M. Cherfi : On a toujours voulu distraire. On mène parallèlement un combat politique, à l'occasion de la double-peine, des sans-papiers, de la venue de J.-M. Le Pen à Toulouse. Là, on rigole pas, on lutte. Mais quand on chante, il faut que ce soit agréable : le travail sur la mélodie, les mots, est fait avec l'objectif d'être agréable. Si notre objectif politique est plus large, une chanson même politique est d'abord une chanson : elle sert à faire du lien, elle fait appel aux sens, c'est sa force.

Mots : La définiriez-vous comme un symbole, au même titre qu'un slogan, un drapeau?

M. Cherfi : Absolument : elle fait signe. Notre travail consiste en partie à rechercher des symboles, on est en quête de symboles.

Mots : Qui se retrouve autour des symboles que vous proposez?

M. Cherfi : Les lilis et les bobos ${ }^{7}$. Zebda fait maintenant partie du magasin politique branché. Et puis nos valeurs sont rassurantes : liberté,

6. Animateur du jeu « Le bigdil » sur TF1.

7. Les « lilis » désignent les « libéraux-libertaires » et les «bobos » les « bourgeoisbohèmes $»$. 
égalité, fraternité, République et francité... On est rassurants pour les progressistes.

Mots : N'est-ce pas un échec?

M. Cherfi : Il ne peut pas en être autrement. Les enfants des quartiers écoutent "Nique ta mère », mais pas nos chansons. Pour eux, on est une bande d'intellos privilégiés par l'existence, des bobos de l'émigration, même si nos parents étaient des prolos. On n'est pas les porte-paroles des enfants de l'immigration; on est d'abord français.

Mots : Et pourtant, le nom même de «Zebda ${ }^{8}$...?

M. Cherfi : C'est de la provoc, ça veut dire « Nous, Français dont vous ne voulez pas ». La France peut être aussi multiculturelle que l'on veut tant que ça l'arrange, mais l'arabité ça ne passe pas.

Mots : Est-ce que vous cherchez à discuter avec ces jeunes, ou est-ce que ce n'est plus votre problème?

M. Cherfi : On essaie d'abord d'être nous-mêmes; les discours radicaux de certains beurs du type "La France est un pays raciste ", " La France est une dictature colonialiste » ne sont pas les nôtres. Nous pensons que la France est avant tout un pays de droit, et cela nous coupe des jeunes des quartiers qui veulent entendre «La France nique notre race, elle nous tue $» .$. . Ce qui est aussi une réalité.

Plus encore que des mots, les jeunes des cités ont besoin d'actes. Quand vous rentrez dans une boite de nuit, quand vous cherchez un appart, un boulot, quand vous allez à la sécu, à l'ANPE, à l'école..., c'est l'arabité qu'on vous renvoie à la figure.

Un enfant issu de l'immigration échappe difficilement à quelque chose de l'ordre de l'échec. Mon identité est un échec; ma langue est un échec; mon nom est un échec; mon pays est un échec; ma religion est un échec : c'est Ben Laden plus que la spiritualité ou le Recteur de la Mosquée de Paris.

Quand J. Chirac fait son discours sur le bruit et l'odeur, il nous fait perdre 30 ans. Pour moi, cette phrase ne passe pas et j'attends toujours des excuses... Les politiques ne sont pas là-dedans, ni J. Chirac, ni L. Jospin,

8. Zebda signifie « beurre » en arabe. 
ni le PC...Les beurs des partis politiques sont les colonisés d'aujourd'hui. Sur les listes électorales, ils figurent dans les dernières places.

Mots : L'album Motivés s'insère-t-il pour vous dans une tradition toulousaine de la chanson contestataire, comme Les Fabulous Troubadors ${ }^{9}$, par exemple?

M. Cherfi : Oui, bien sûr : on s'inscrit dans une dimension toulousaine, nationale et internationale de la chanson contestataire, et l'idée d'appartenir au terroir toulousain nous émeut... Mais moi, j'aspire à la francité, pour moi c'est un aboutissement, et c'est pas ce que peuvent défendre les Fabulous Troubadors!

Mots : A partir de quand estimez-vous qu'une chanson est politique? Considérez-vous, dans votre "production », qu'il y a des albums politiques et d'autres qui ne le sont pas?

M. Cherfi : Pour moi, tout ce que j'écris est politique.

Mots : " Tomber la chemise » est une chanson politique? Quel est le statut d'une chanson comme ça par rapport à votre engagement politique?

M. Cherfi : C'est probablement la moins politique, mais j'y dis quand même : « Ce que la colère a fait de meilleur », j'y parle des « enfants de la cité ». Comme je l'ai dit, on est à la fois porteur d'un message politique et d'une nécessaire distraction. Il s'agit d'intégrer la gaité dans le combat contre le racisme, le fascisme, contre toutes ces intolérances. D'où la présence à la fois d'une chanson comme « Le bruit et l'odeur» et comme « Tomber la chemise ».

Mots : D'autres groupes vous paraissent-ils incarner aujourd'hui la chanson politique contemporaine?

M. Cherfi : Il y a une tradition classique : Renaud, Lavilliers, Noir Désir, Manu Chao. Certains artistes sont obsédés par l'idée de l'injustice sociale et ne peuvent bâtir leur histoire artistique qu'en établissant ce lien. Mais certains ne recherchent qu'une caution, comme les Enfoirés par exemple : des « stars » qui en fait n'en ont rien à faire de tout ça et qui se

9. Groupe musical toulousain occitano-gauchiste dont le leader, Claude Sicre, milite au quotidien dans le quartier métissé d'Arnaud Bernard. 
rachètent une âme. Il y a ceux qui ont une vraie conscience politique et ceux qui ont une mauvaise conscience politique...

Mots : Quelles différences feriez-vous entre Manu Chao, Noir Désir, Lavilliers, Renaud, entre ces chanteurs que vous reconnaissez comme authentiquement engagés?

M. Cherfi : Manu Chao mène un combat sincère, il est vécu comme une sorte de Che musical et artistique, un peu romanesque... Pour Noir Désir, les choses sont plus intellectualisées : ils s'attachent à défendre les sans-papiers, ce qui est un combat très difficile, très ingrat; défendre les pauvres, c'est bien plus facile. Lavilliers est sur un registre ouvrier, Renaud prône une forme de subversion bourgeoise.

Mots : Pour revenir à l'album Motivés, on a déjà souligné la grande diversité des chansons qui y figurent : comment définir une chanson politique? Qu'est-ce qui fait qu'une chanson comme "Le temps des cerises" par exemple soit vécue comme "politique »? Qu'est-ce qui fédère tout ça, quel serait le fil rouge - bien sûr - qui permettrait de relier toutes ces chansons?

M. Cherfi : Le fil est ténu; mais portés par des gens qui ont «l'image de », ces chants signifient la lutte, le combat anti-bourgeois, anticapitaliste. Motivés a été chantée dans la rue par des manifestants de tous bords, c'est une chanson qui donne le moral, qui donne envie d'aller plus loin, de continuer le combat. Et si elle n'est que ça, c'est déjà pas mal. 